\title{
Development and validation of the Adolescent Asthma Self-Efficacy Questionnaire (AASEQ)
}

\author{
Simone Holley ${ }^{1,10}$, Rebecca Knibb ${ }^{2,10}$, Sue Latter ${ }^{3}$, Christina Liossi ${ }^{4,5}$, \\ Frances Mitchell ${ }^{6}$, Ruth Radley ${ }^{7,8}$ and Graham Roberts ${ }^{1,6,9}$
}

Affiliations: ${ }^{1}$ Clinical and Experimental Sciences and Human Development in Health, University of Southampton, Southampton, UK. ${ }^{2}$ Dept of Psychology, Aston University, Birmingham, UK. ${ }^{3}$ School of Health Sciences, University of Southampton, Southampton, UK. ${ }^{4}$ School of Psychology, University of Southampton, Southampton, UK. ${ }^{5}$ Dept of Paediatric Psychology, Great Ormond Street Hospital for Children NHS Foundation Trust, London, UK. ${ }^{6}$ The David Hide Asthma and Allergy Research Centre, St Mary's Hospital, Isle of Wight, UK. ${ }^{7}$ Dept of Paediatrics, Salisbury NHS Foundation Trust, Salisbury, UK. ${ }^{8}$ NIHR/Wellcome Trust Clinical Research Facility, University Hospital Southampton NHS Foundation Trust, Southampton, UK. ${ }^{9}$ NIHR Southampton Biomedical Research Centre, University Hospital Southampton NHS Foundation Trust, Southampton, UK. ${ }^{10}$ Both authors contributed equally.

Correspondence: Graham Roberts, Paediatric Allergy and Respiratory Medicine (Mailpoint 803), Southampton University Hospital NHS Foundation Trust, Tremona Road, Southampton, S016 6YD, UK.

E-mail: g.c.robertsdsoton.ac.uk

@ERSpublications

The AASEQ is a reliable and valid tool that is likely to aid future understanding of adolescent asthma self-management and be a useful surrogate end-point to assess the impact of behavioural interventions. https://bit.ly/2DmL5KI

Cite this article as: Holley S, Knibb R, Latter S, et al. Development and validation of the Adolescent Asthma Self-Efficacy Questionnaire (AASEQ). Eur Respir J 2019; 54: 1801375 [https://doi.org/10.1183/ 13993003.01375-2018].

ABSTRACT Perceived self-efficacy is the belief that one can manage prospective situations. Good asthma self-management self-efficacy is associated with better asthma outcomes. However, a well-developed and validated tool to measure adolescent asthma self-management self-efficacy is lacking. Our objective was to develop and validate an Adolescent Asthma Self-Efficacy Questionnaire (AASEQ).

The first stage of the study included a review of the literature, interviews with adolescents with asthma and consultations with parents and relevant healthcare professionals to develop a prototype scale. To assess reliability and validity, a further group of adolescents completed the prototype scale, the General Self-Efficacy Scale and KidCOPE (measures coping styles). Retesting was undertaken to assess longitudinal validity.

Interviews with 28 adolescents and consultations with other stakeholders resulted in a 38-item prototype scale. Key themes were medication, symptom management, triggers, knowledge, attitude and beliefs around asthma, supportive relationships, schools and healthcare professionals. The prototype scale was completed by 243 adolescents. Factor and reliability analysis reduced it to a 27 -item scale with four subsections: symptom management; medication; friends, family and school; and asthma beliefs. The 27-item scale had respectable to excellent internal consistency ( $\alpha$ 's $0.78-0.91$ ) with results that were stable over time (intra-class correlation $=0.82$ ) in 63 subjects who completed it twice. Better adolescent asthma self-efficacy was associated with better general self-efficacy and indices of better asthma management.

The AASEQ is a reliable and valid tool that is likely to aid future research and practice focused on adolescent asthma self-management and could be a useful intermediate outcome measure to assess the impact of behavioural interventions.

This article has supplementary material available from erj.ersjournals.com

Received: July 202018 | Accepted after revision: April 092019

Copyright OERS 2019 


\section{Introduction}

Many adolescents with asthma have suboptimal disease control despite the availability of effective therapies [1]. For some, poor asthma control will be a consequence of suboptimal self-management, particularly adherence to treatment [2]. Research has identified several psychosocial and behavioural factors that influence asthma self-management in adolescence, e.g. forgetting treatment, lack of knowledge about asthma and treatments, treatment burden, erroneous beliefs, embarrassment at having asthma and communication difficulties with healthcare practitioners [3].

Self-management self-efficacy in chronic disease is an important concept [4]. Perceived self-efficacy is defined as "the belief in one's capabilities to organise and execute the courses of action required to manage prospective situations" [5]. Improving self-efficacy can lead to individuals feeling more confident to master challenging problems, developing a stronger sense of commitment to dealing with tasks and not feeling that situations are beyond their capabilities [5]. In asthma, self-management self-efficacy would cover strategies to prevent symptoms including the use of preventers and preparation to manage symptoms as well as managing them. Good asthma self-efficacy has been found to be associated with better asthma outcomes, including less hospitalisation [6-10]. In addition, there is some evidence that interventions designed to improve self-efficacy may improve asthma outcomes [11].

A child asthma self-efficacy measure exists that was developed in the United States for children aged 7-15 years [12]. Studies using this measure, to explore the role of self-efficacy in adolescents with asthma, have reported inconclusive results. RHEE et al. [13] found that self-efficacy predicted barriers to self-management, such as poor relationships with healthcare professionals, negative perceptions to medication and difficulties with adherence. Meanwhile, Sleath et al. [14] and Zebracki and Drotar [15] found that asthma self-efficacy was associated with better adherence to asthma medications in adolescents. In contrast, Zebracki and Drotar. [15] and Riekert et al. [16] found that better self-efficacy was not associated with improvements in other aspects of self-management. Improvements in self-efficacy have been shown in intervention studies following the use of a mobile asthma action plan [17] and peer-led education [18], but not following the use of a motivational interviewing intervention [16].

A number of other groups have examined self-efficacy in asthma using other approaches. van DeLLen et al. [19] reported that higher self-efficacy was associated with better adherence with self-efficacy measured using a single question "How difficult will it be for you to take your ICSs [inhaled corticosteroids] on a daily basis in the near future?". VAN Es and co-workers $[20,21]$ used a short questionnaire to measure self-efficacy and did not find any improvements following an intervention programme.

One possible reason for the equivocal results found here is the way in which the child asthma self-efficacy scale was developed. Patient-reported outcome measures should be developed and validated using rigorous and established methods that establish content validity and reliability. Preliminary qualitative work using open-ended questions should be used to gain a meaningful perspective with adolescents with asthma as the population of interest [22]. Self-efficacy instruments in particular need to identify the challenges that people face to perform activities; questions should be formulated to include a judgment of perceived capability ("I can do") for carrying out specific activities; and the measurement scale should ideally range from 0 to $100[23,24]$.

The asthma self-efficacy measure developed by BuRSCH et al. [12] used structured interviews rather than semi-structured ones. There is also a lack of information regarding how scale items were selected, whether their construction involved adolescents, and test-retest reliability data. In addition, the measure may not be appropriate for use with adolescents, given it was developed for children aged 8-17 years; adolescents have a very different experience to younger children [25]. A further adolescent asthma self-efficacy questionnaire was developed 25 years ago in the Netherlands with participants aged 10-18 years [26]. It has similar methodological limitations (reviewed in FreI et al. [27]).

In this study we describe the initial development and validation of a new measure, the Adolescent Asthma Self-Efficacy Questionnaire (AASEQ). This has been developed for use with adolescents aged 12-18 years following contemporary scale guidelines and focusing on asthma self-management self-efficacy.

\section{Methods}

Ethical approval was provided by the National Health Service ethics committees (supplementary material). All participants and parents/carers gave informed consent.

\section{Item generation}

Participants and procedures

This phase was conducted between October 2014 and March 2015 in the south of England. Participants were aged 12-18 years with doctor-diagnosed asthma (as coded in medical notes), prescribed regular 
prophylactic asthma medication and with no other significant long-term medical condition (apart from hay fever, eczema or food allergy), recruited from three general practices and two hospitals. Purposive sampling was used to ensure that participants ranging in age, sex and asthma control were included. In addition, a number of their parents and healthcare professionals were consulted (supplementary material).

Interviews and analysis

Interviews and focus groups were conducted by a psychologist (SH), not previously known to the participants, with experience in conducting focus groups and interviews with adolescents. They were audiotaped and transcribed verbatim, then analysed by inductive thematic analysis [28]; further details are included in the supplementary material. The multidisciplinary group of authors used the themes from the initial analysis and the literature review to form items for inclusion in a prototype questionnaire. Feedback on the items and rating scale was then sought from parents and healthcare practitioners working with adolescents with asthma (including four paediatric consultants with an interest in respiratory disease and three paediatric asthma nurses). Six adolescent participants who had taken part in the qualitative interviews reviewed the prototype questionnaire to check that items and the rating scale were understandable; no changes were deemed necessary. Based on guidelines provided by BANDURA [23], items were worded as statements with a rating scale of $0-100$, where participants are asked to rate how confident they are that they could do each item, with 0 being cannot do at all, 50 being moderately can do and 100 being highly certain can do. This process resulted in a 38-item prototype AASEQ.

\section{Scale reliability and validity \\ Participants and procedure}

To assess reliability and validity of the scale in a large sample, participants with asthma were recruited from the general population to take part in an online questionnaire. Recruitment took place between July 2015 and June 2016. A convenience sample was recruited from 19 hospital outpatients and eight primary care general practitioner centres across England. Asthma UK and the Anaphylaxis Campaign advertised the study through social media outlets (Facebook and Twitter) and newsletters. Participants were provided with information about the study and a secure internet address where they could access the questionnaire after completing an online consent. Participants were informed that on completion of the questionnaire they would be entered into a prize draw to win a gift voucher (one prize of $£ 50$, five runner-up prizes of $£ 10$ ). The inclusion criteria were age $12-18$ years with doctor-diagnosed asthma and no other chronic illness that has a major impact on daily life (apart from hay fever, eczema and food and animal allergy, given the high level of comorbidity of these with asthma). Participants could complete the questionnaire anonymously, although email addresses were requested in order to conduct the AASEQ repeat test.

\section{Cross-sectional validation measures}

Adolescents completed two scales to assess convergent construct validity: the KidCOPE [29] and the Generalized Self-Efficacy Scale (GSES) [30]. These scales are well-used; have excellent reliability and validity for the age range of our participants; are quick to complete; and measure constructs we hypothesised would correlate with the AASEQ. Further details are given in the supplementary material. In addition, adolescents provided details about their asthma such as length of time since diagnosis, triggers, medication and number of hospital admissions due to asthma. They were also asked to rate how often they forgot their preventer inhaler on a six-point scale from never to always.

\section{Consistency over time}

Participants were sent an email asking them to repeat the AASEQ 4 weeks after completing the baseline questionnaire. They were asked whether they had experienced any asthma-related events during that time interval.

\section{Statistical analysis}

Data analyses were conducted using SPSS (version 22; IBM, Armonk, NY, USA); missing data was treated listwise. Standard analysis to explore reliability and validity was then applied [31]. Principal components analysis was conducted to shorten the questionnaire to remove redundancy. Cronbach's $\alpha$ coefficient and Guttman's split-half coefficient were conducted to assess internal reliability of the scale. Agreement with other validated questionnaires (construct validity) was assessed using Pearson's bivariate correlations. Consistency of the questionnaire over time (test-retest reliability) was assessed using intra-class correlation (ICC). All tests were two-tailed with a significance level set at $\mathrm{p}<0.05$. Further details are available in the supplementary material. 


\section{Results}

Item generation

28 adolescents aged 12-18 years with doctor-diagnosed asthma participated. Six adolescents took part in one focus group and 22 adolescents took part in a 1:1 interview. Full details of this qualitative phase (supplementary table S1), item generation and prototype AASEQ scale (supplementary box S1) development can be found in the online supplement.

Scale reliability and validity

A total of 243 participants completed the baseline questionnaires. Demographic information and asthma characteristics of these participants can be found in table 1. Three participants did not complete the AASEQ and were removed from analysis to assess scale reliability and validity. There were only 36 missing

\section{TABLE 1 Demographic information and asthma characteristics of participants}

\begin{tabular}{|c|c|c|}
\hline & Baseline & Retest \\
\hline Subjects n & 243 & 63 \\
\hline Age years & $14.6 \pm 1.8$ & $14.8 \pm 1.9$ \\
\hline Age range years & $12-18$ & $12-18$ \\
\hline Age of onset of asthma years & $4.8 \pm 4.2$ & \\
\hline Length of time since diagnosis years & $9.8 \pm 4.3$ & \\
\hline \multicolumn{3}{|l|}{ Sex } \\
\hline Male & 97 (39.9) & $16(25.4)$ \\
\hline Female & $146(60.1)$ & $47(74.6)$ \\
\hline \multicolumn{3}{|l|}{ Ethnicity } \\
\hline White British & 206 (84.8) & 57 (90.5) \\
\hline \multicolumn{3}{|l|}{ Managed by } \\
\hline Primary care & $102(42.0)$ & $32(50.8)$ \\
\hline Secondary care & $139(57.2)$ & $31(49.2)$ \\
\hline \multicolumn{3}{|l|}{ Recruited from } \\
\hline Hospital & $184(75.7)$ & $41(65.1)$ \\
\hline GP & 23 (9.5) & $8(12.7)$ \\
\hline Social media & $34(14.0)$ & $14(22.2)$ \\
\hline \multicolumn{3}{|l|}{ Self-reported asthma triggers } \\
\hline Weather & $187(77.0)$ & \\
\hline Pollen & $161(66.3)$ & \\
\hline Emotions & $164(67.5)$ & \\
\hline Fumes & $136(56.0)$ & \\
\hline \multicolumn{3}{|l|}{ Self-reported asthma triggers } \\
\hline Dust & $73(30.0)$ & \\
\hline Pets & $140(57.6)$ & \\
\hline Colds or flu & $42(17.3)$ & \\
\hline Cigarette smoke & $118(48.6)$ & \\
\hline Food or drinks & $206(84.8)$ & \\
\hline Soaps/sprays & $147(60.5)$ & \\
\hline \multicolumn{3}{|l|}{ Self-report of forgetting preventer medication } \\
\hline Never & 59 (24.3) & \\
\hline Occasionally & $95(39.1)$ & \\
\hline Once a week & $21(8.6)$ & \\
\hline Half the time & $20(8.2)$ & \\
\hline Most of the time & 28 (11.5) & \\
\hline All the time & $18(7.4)$ & \\
\hline Number of asthma exacerbations in last year ${ }^{\#}$ & $3.5 \pm 5.0$ & \\
\hline Number of oral corticosteroid courses in last year" & $3.2 \pm 5.4$ & \\
\hline Total number of hospital visits due to asthma & $7.7 \pm 20.3$ & \\
\hline \multicolumn{3}{|l|}{ Other allergic disease } \\
\hline Eczema & $107(44)$ & \\
\hline Hay fever & $187(77)$ & \\
\hline Food allergy & $66(27.2)$ & \\
\hline Animal allergy & $115(47.30)$ & \\
\hline
\end{tabular}

Data are presented as mean \pm SD or $\mathrm{n}(\%)$, unless otherwise stated. GP: general practitioner. " : "how many asthma exacerbations did you have last year?"; ": "how many courses of steroid (prednisolone) did you need in the last year?". 
items across the whole dataset for the AASEQ ( $n=9234$ data points). Details of missing data and floor and ceiling effects are shown in the online supplementary material (figure S1).

\section{Internal structural validity of the AASEQ}

Principal components analysis with a varimax rotation was conducted on the 38 items of the prototype AASEQ (supplementary box S1). Four items with low factor loadings were removed, giving a 34-item solution which explained $58.3 \%$ of the total variance in the data. A clear interpretation of the factors could be made and factors were called friends, family and school; symptom management; asthma beliefs; and medication (table 2). Further details are listed in the supplementary material.

\section{Internal reliability of the AASEQ}

The 34 items had excellent internal consistency (table 3). On inspection of the items, it was felt that some were very similar, for example, items such as "talking to teachers" and "talking honestly to teachers" were originally included in the scale to see which item was a more reliable indicator of self-efficacy. As these items contributed equally well in the analysis it was felt that the scale could be made more parsimonious

\section{TABLE 2 Factor analysis of the Adolescent Asthma Self-Efficacy Questionnaire 34-item scale}

Factor 1

Factor 2 Factor 3

Factor 4

\section{Friends, family and school}

I can talk honestly to my teachers about my asthma

I can talk to my teachers about my asthma"

I can talk honestly to my friends about my asthma

I can talk to my friends about my asthma\#

I can take my inhalers in front of my friends

I can take my inhalers around other people at school

I can ask my teachers for help if I am having trouble breathing or having an asthma attack

I can ask my friends for help if I am having trouble breathing or having an asthma attack

I can talk honestly to my doctor or nurse about my asthma

I can talk to my doctor or nurse about my asthma ${ }^{\#}$

I can talk honestly to my parents about my asthma

I can talk to my parents about my asthma

I can ask my parents for help if I am having trouble breathing or having an asthma attack

\section{Symptom management}

I know how to stay calm when I am having trouble breathing

I know how to control my asthma when I am having trouble breathing

I can stay calm when I am having trouble breathing ${ }^{\#}$

I can be prepared to deal with an asthma attack

I know when to use my inhaler to manage a serious breathing problem

I know what to do to avoid triggers for my asthma

I know when I might need to go to hospital because of a serious breathing problem

I know when I am out of breath because of my asthma rather than because I feel a bit panicky

I know when I am out of breath because of my asthma rather than because of exercise

I can have my medication with me at all times ${ }^{\#}$

\section{Asthma beliefs}

I can do the things that I want to do

I can have a normal life

I can control my asthma day-to-day

I can do physical activity such as sports

I am in control of my asthma

\section{Medication}

I know what my preventer inhaler is for

I know what my reliever inhaler is for

I know what my inhalers are for ${ }^{\#}$

I know which of my inhalers I need to take

I know when to use my asthma medication

I know how to correctly use my asthma inhaler/spacer/medication

\section{Eigenvalues}

Percentage variance explained

\begin{tabular}{|c|c|c|c|}
\hline 0.83 & 0.19 & -0.01 & -0.04 \\
\hline 0.82 & 0.18 & 0.06 & 0.03 \\
\hline 0.81 & 0.07 & 0.20 & 0.14 \\
\hline 0.81 & 0.05 & 0.16 & 0.09 \\
\hline 0.75 & 0.20 & 0.16 & 0.04 \\
\hline 0.74 & 0.26 & 0.07 & -0.02 \\
\hline 0.73 & 0.14 & -0.002 & 0.04 \\
\hline 0.69 & 0.06 & 0.17 & 0.10 \\
\hline 0.69 & 0.15 & -0.02 & 0.05 \\
\hline 0.66 & 0.15 & 0.01 & 0.08 \\
\hline 0.65 & -0.12 & 0.35 & 0.17 \\
\hline 0.61 & -0.14 & 0.33 & 0.20 \\
\hline 0.56 & -0.13 & 0.21 & 0.27 \\
\hline 0.03 & 0.81 & 0.22 & 0.07 \\
\hline 0.07 & 0.78 & 0.18 & 0.20 \\
\hline 0.06 & 0.76 & 0.28 & 0.003 \\
\hline 0.06 & 0.69 & 0.12 & 0.32 \\
\hline 0.05 & 0.64 & 0.06 & 0.41 \\
\hline 0.04 & 0.60 & 0.12 & 0.22 \\
\hline 0.19 & 0.56 & 0.01 & 0.34 \\
\hline 0.27 & 0.53 & 0.04 & 0.24 \\
\hline 0.36 & 0.52 & 0.02 & 0.21 \\
\hline 0.16 & 0.43 & -0.22 & 0.17 \\
\hline 0.17 & 0.05 & 0.88 & -0.02 \\
\hline 0.20 & -0.001 & 0.87 & -0.05 \\
\hline 0.08 & 0.28 & 0.83 & -0.004 \\
\hline 0.23 & 0.15 & 0.74 & -0.03 \\
\hline 0.14 & 0.29 & 0.69 & 0.03 \\
\hline 0.02 & 0.21 & -0.01 & 0.80 \\
\hline 0.01 & 0.18 & -0.05 & 0.79 \\
\hline 0.12 & 0.26 & -0.03 & 0.75 \\
\hline 0.08 & 0.24 & 0.01 & 0.69 \\
\hline 0.18 & 0.37 & -0.02 & 0.52 \\
\hline 0.24 & 0.21 & 0.02 & 0.46 \\
\hline 7.35 & 5.00 & 3.89 & 3.60 \\
\hline 21.61 & 14.71 & 11.44 & 10.57 \\
\hline
\end{tabular}

Data are presented as the factor loading for each question for each of the four factors, unless otherwise stated. Bold type indicates which factor each question loads onto in the principal component analysis. An eigenvalue of $>1$ indicates that a factor is important. \#: items removed to create a more parsimonious 27 -item scale. 
by the removal of the item with the lower factor loading (indicated by ${ }^{\#}$ in table 2 ) resulting in a 27 -item scale (box 1). This did not affect the reliability of the scale substantially (table 3 ). All AASEQ answers are summed and then divided by 27 to get a total mean score (0-100). Subscale items are summed and divided by the number of items in each subscale. A higher score indicates greater self-efficacy for management of asthma.

\section{Cross-sectional validity of AASEQ}

The total AASEQ score significantly correlated with total general self-efficacy with greater asthma management self-efficacy associated with greater general self-efficacy. Each subscale of the AASEQ significantly correlated with the GSES (table 4). In relation to coping, the total AASEQ score and all the subscales had small- to medium-sized positive correlations with problem-solving coping, indicating that greater use of this coping strategy related to greater asthma self-efficacy (table 4).

Greater asthma management self-efficacy (total score and all subscales apart from symptom management) significantly related to less use of social withdrawal, with small- to medium-sized correlations. Poorer asthma management self-efficacy for the family, friends and school subscale was associated with greater use of blaming others (medium-sized correlation) and less use of social support as a way of coping (small correlation). Better symptom management and medication self-efficacy related to greater use of cognitive restructuring as a way of coping (table 4). Together these results demonstrate good construct validity of the AASEQ compared to general self-efficacy and moderate construct validity compared to general coping styles.

The relationship between markers of poor asthma control and the AASEQ showed small- to mediumsized negative correlations (table 5). A greater number of self-reported asthma exacerbations in the past year was significantly associated with poorer total asthma self-efficacy, asthma beliefs and use of friends, family and school. More self-reported use of oral corticosteroids in the past year and more self-reported hospital visits for asthma were associated with poorer asthma beliefs, but a belief in better symptom management, demonstrating that adolescents knew what to do if they had an asthma attack or needed to go to hospital, but felt that they were not able to have a normal life or be in control of their asthma.

Factors likely to be related to self-management were also examined (table 5). A greater frequency in forgetting their preventer inhaler significantly correlated with lower total asthma self-efficacy scores and lower subscale scores for friends, family and school, symptom management and use of medication. Having asthma for a longer duration was significantly correlated with better asthma symptom management. Poorer asthma self-efficacy also related to having comorbid hay fever and food allergy (supplementary material).

\section{Consistency over time of the AASEQ}

183 participants were sent an email asking them to complete the AASEQ for a second time. Of these, 63 (34.4\%) responded to the request to complete the retest. There were no differences in responders except that they reported forgetting their preventer inhaler more often (mean \pm SD $3.00 \pm 1.69$ versus $2.53 \pm 1.52, \mathrm{p}<0.05$ ).

There was a strong ICC of 0.82 between the baseline total scale score and the retest total scale score (table 3

TABLE 3 Internal consistency and consistency over time of the 27-item Adolescent Asthma Self-Efficacy Questionnaire (AASEQ) scale and subscales

All repeat participants

$\begin{array}{cc}\text { Initial } & \begin{array}{c}\text { Repeat } \\ \text { assessment }\end{array}\end{array}$

Only repeat participants reporting no change

\begin{tabular}{cc}
\hline Initial & $\begin{array}{c}\text { Repeat } \\
\text { assessment } \\
\text { assessment }\end{array}$
\end{tabular}

Cronbach's $\alpha$

34-item 27-item

ICCs for test-retest

\begin{tabular}{cc}
\hline All retest & Participants \\
participants & $\begin{array}{c}\text { reporting no } \\
\text { change }\end{array}$
\end{tabular}

\begin{tabular}{|c|c|c|c|c|c|c|c|c|}
\hline Subjects $\mathrm{n}$ & \multicolumn{2}{|c|}{63} & \multicolumn{2}{|c|}{22} & \multirow[b]{2}{*}{0.92} & \multirow[b]{2}{*}{0.91} & \multirow{2}{*}{$\begin{array}{c}63 \\
0.82\end{array}$} & \multirow{2}{*}{$\begin{array}{c}22 \\
0.81\end{array}$} \\
\hline Total scale & $82.32 \pm 12.67$ & $82.92 \pm 14.24$ & $83.27 \pm 11.29$ & $85.13 \pm 10.52$ & & & & \\
\hline \multicolumn{9}{|l|}{ Subscales } \\
\hline $\begin{array}{c}\text { Friends, family } \\
\text { and school }\end{array}$ & $86.46 \pm 17.27$ & $82.55 \pm 21.66$ & $88.02 \pm 12.04$ & $86.95 \pm 12.89$ & 0.93 & 0.90 & 0.90 & 0.72 \\
\hline Symptom management & $76.22 \pm 18.36$ & $77.92 \pm 18.23$ & $70.74 \pm 17.32$ & $75.82 \pm 18.45$ & 0.88 & 0.87 & 0.65 & 0.58 \\
\hline Asthma beliefs & $77.33 \pm 22.17$ & $82.05 \pm 20.15$ & $90.94 \pm 10.44$ & $90.21 \pm 11.94$ & 0.90 & 0.90 & 0.90 & 0.72 \\
\hline Medication & $90.35 \pm 12.25$ & $92.48 \pm 8.56$ & $87.14 \pm 12.77$ & $91.68 \pm 9.85$ & 0.84 & 0.78 & 0.58 & 0.64 \\
\hline
\end{tabular}

Data are presented as mean $\pm \mathrm{SD}$, unless otherwise stated. Cronbach's $\alpha$ represents the consistency of the questionnaire and the subscales. Intra-class correlations (ICCS) represent the consistency of the questionnaire over time (test-retest reliability). No change related to having not experienced an asthma attack; not being admitted to hospital due to their asthma; not being seen a healthcare professional for their asthma; no change in asthma medication; and having had no new asthma education since completing the initial questionnaire. 


\section{BOX 1 27-item final Adolescent Asthma Self-Efficacy Questionnaire}

This questionnaire is designed to help us get a better understanding of how you manage your asthma. Please rate how certain you are that you can do each of the things described below by writing the appropriate number

For each of the following statements, rate how confident you feel by choosing a number from 0 to 100 using the scale given below:

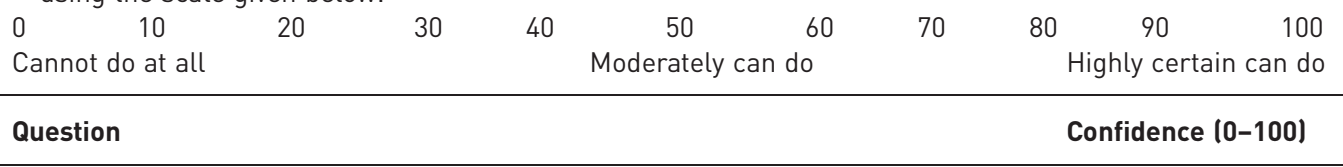

\section{Medication}

I am confident that:

I know how to correctly use my asthma inhaler/spacer/medication

I know when to use my asthma medication

I know which of my inhalers I need to take

I know what my preventer inhaler is for

I know what my reliever inhaler is for

\section{Symptom management}

I am confident that:

I can be prepared to deal with an asthma attack

I know how to stay calm when I am having trouble breathing

I know when I am out of breath because of my asthma rather than because of exercise

I know when I am out of breath because of my asthma rather than because I feel a bit panicky

I know how to control my asthma when I am having trouble breathing

I know when to use my inhaler to manage a serious breathing problem

I know when I might need to go to hospital because of a serious breathing problem

I know what to do to avoid triggers for my asthma

\section{Asthma beliefs}

I am confident that:

I am in control of my asthma

I can do physical activity such as sports

I can have a normal life

I can do the things that I want to do

I can control my asthma day-to-day

\section{Friends, family and school}

I am confident that:

I can take my inhalers in front of my friends

I can take my inhalers around other people at school

I can talk honestly to my friends about my asthma

I can talk honestly to my parents about my asthma

I can talk honestly to my doctor or nurse about my asthma

I can talk honestly to my teachers about my asthma

I can ask my parents for help if I am having trouble breathing or having an asthma attack

I can ask my teachers for help if I am having trouble breathing or having an asthma attack

I can ask my friends for help if I am having trouble breathing or having an asthma attack

for total and subscale ICCs). Adolescents reporting no change in their asthma $(n=22)$ had similar results (ICC 0.81; table 3).

\section{Discussion}

The AASEQ (box 1) is the first asthma self-efficacy scale developed specifically for adolescents aged 12-18 years using recommended and robust scientific methods [23]. Previous scales for measuring adolescent asthma self-efficacy $[12,26]$ have lacked rigorous development processes such as a systematic literature search, adequate inclusion of stakeholder opinion, test-retest reliability and construct validity [27]. In contrast, we conducted a comprehensive literature search, interviewed several stakeholder groups (adolescents, their parents and their healthcare professionals), and established test-retest reliability and construct validity. 
TABLE 4 Pearson's correlations between the Adolescent Asthma Self-Efficacy Questionnaire (AASEQ) scale and subscales, the Generalized Self-Efficacy Scale (GSES) and the KidCOPE

\section{AASEQ total AASEQ subscales}

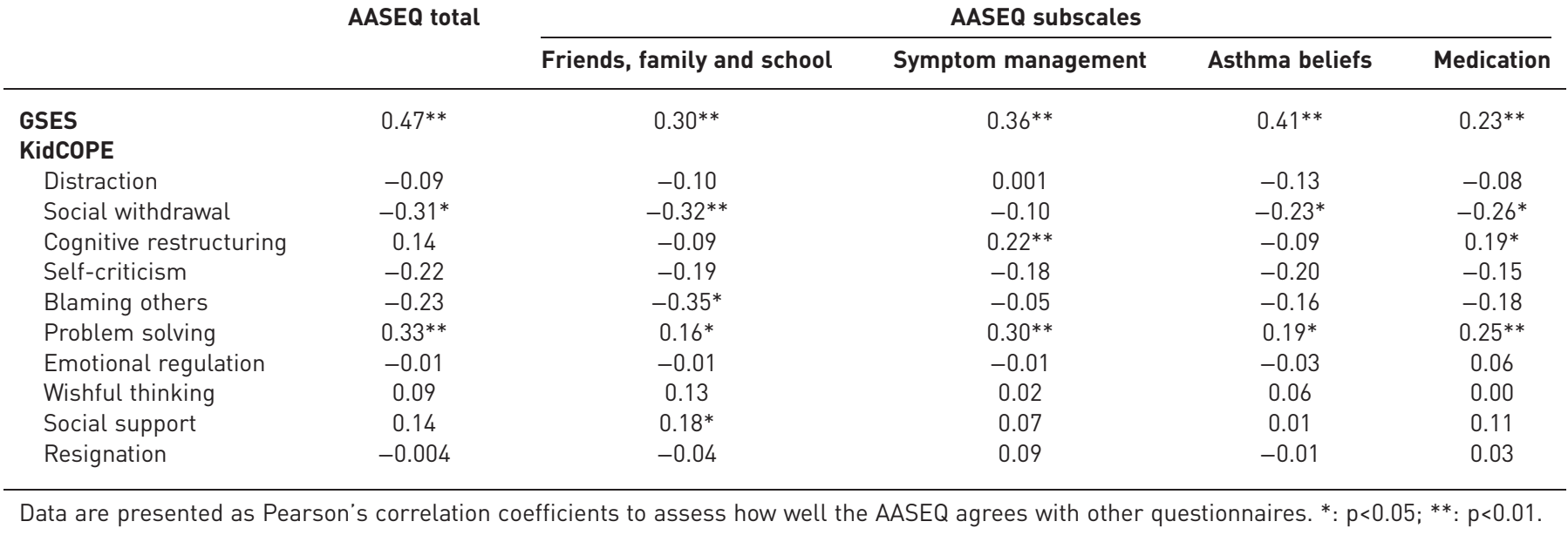

As an example, the AASEQ correlates with markers of asthma control. So the AASEQ focuses on the specific challenges that this group face in developing their independent self-management skills [32].

The overall AASEQ scale and all subscales demonstrate good to excellent internal reliability and stability over time. In the test-retest assessment, scores for self-efficacy for asthma medication increased slightly from time one to time two; it may be that completing the scale at time one prompted adolescents to think about and consequently remember information about their asthma medication, resulting in them reporting more confidence in using it when completing the scale again. Finally, the scale has good construct validity, as demonstrated by how it correlated with the GSES and the KidCOPE (further discussed in the supplementary material).

A strength of this study is the large sample of adolescents who completed the scale, enabling a range of validity analyses to be conducted. The majority were recruited from primary or secondary care, ensuring that the analysis was not completely reliant on self-report of an asthma diagnosis.

There are a number of limitations that need to be addressed in further work. Although we piloted the scale with adolescents, we did not conduct cognitive interviews and these would be useful to check understanding of the items and the response scale. Although not identified as an issue by adolescents, the scale instructions could be further tested. Confirmatory factor analysis is needed, which will enable us to see if the subscales found in the exploratory factor analysis reported here can be replicated. Although a

TABLE 5 Pearson's correlations between the Adolescent Asthma Self-Efficacy Questionnaire (AASEQ) scale and subscales, asthma control and factors that could affect asthma self-management

\section{AASEQ total}

AASEQ subscales

\begin{tabular}{cccc}
\hline $\begin{array}{c}\text { Friends, family } \\
\text { and school }\end{array}$ & $\begin{array}{c}\text { Symptom } \\
\text { management }\end{array}$ & Asthma beliefs & Medication
\end{tabular}

\section{Asthma control}

Asthma exacerbations in past year $n$

Corticosteroid courses in past year $n$

Hospital visits ever $n$

\section{Self-management}

Forgetting of preventer inhaler $n$

Length of time since diagnosis years

Age of onset of asthma years

Age at completion of the questionnaire years

$-0.19 * *(224)$
$-0.01(217)$
$-0.07(228)$
$-0.23 * *(227)$
$0.13(227)$
$-0.18 * *(227)$
$-0.12(228)$

$-0.17^{*}(230)$
$-0.01(223)$
$-0.03(234)$
$-0.16^{* *}(232)$
$0.07(233)$
$0.15^{*}(233)$
$-0.17^{* *}(234)$

$$
\begin{gathered}
-0.43^{* * *}(236) \\
-0.37 * *(227) \\
-0.14 *(240) \\
0.05(238) \\
-0.03(239) \\
-0.02(239) \\
-0.10(240)
\end{gathered}
$$

Data are presented as Pearson's correlation coefficients $(n)$ to assess how well the AASEQ agrees with asthma parameters. *: $p<0.05$; $* *: p<0.01 ; * * *: p<0.001$. 
large number of participants were included in the development phase, it is possible that a different structure would be apparent with an even larger number of participants. It would also be useful to explore whether greater self-efficacy reported by adolescents relates to better asthma management using more objective indices of asthma control and adherence to medication. The validation work thus far has relied on self-report from adolescents. The ability of the scale to measure change over time in response to an intervention to improve asthma management self-efficacy needs to be ascertained and this is work currently being conducted by the authors. Further work to assess the test-retest reliability, given the small number completing the retest, would be valuable to provide further evidence for the consistency of the tool over time. It is possible that not all the participants had asthma, as $14 \%$ were recruited via social media, although they reported a doctor's diagnosis of asthma. There is the likelihood of a selection bias in our sample, as the most motivated adolescents (who may be more likely to self-manage asthma better) were probably most likely to participate in the survey.

We endeavoured to create a scale that could be used in both research and clinical practice. While taking only 5-10 min to complete, an even shorter version of the scale may be valuable for use in clinical settings when time pressures may prevent the completion of a longer scale. The scale is self-administered and was developed to be understandable to the majority of adolescents aged 12-18 years and completed with little or no input from parents or other adults. We would suggest that adolescent patients could complete the AASEQ scale prior to a clinic consultation to highlight areas where they may most need support with self-management. Healthcare practitioners could then use this information to inform the areas covered in the consultation, ensuring that it focuses on the needs of the adolescent. With self-efficacy being an important issue in long-term conditions [4], we would suggest that the AASEQ scale could be useful in clinical research focused on understanding or improving self-management skills in adolescents with asthma.

In conclusion, the AASEQ is a reliable and valid tool to use with adolescents with asthma and further work on responsiveness of the scale to interventions and validity in relation to objective measures of asthma management should now be conducted. With self-efficacy being an important in the management of long-term conditions, the AASEQ should be useful in assessing adolescent asthma self-management. It should be a useful surrogate end-point to assess the impact of interventions designed to optimise asthma self-management $[16,21]$. Healthcare practitioners, researchers and educators working with this patient group may find this tool useful as an aid to identifying areas in which adolescents are less confident in their asthma management in order to guide specific asthma management education and advice.

Acknowledgements: The study team would like to thank Asthma UK (the Joanna Martin Project) for funding and ongoing support with this study. We would also like to thank the trial steering group for their advice, in particular Mike Thomas, Gary Connett, Hans Michael Haitchi, Woolf Walker, Arvind Nagra, Julian Legg and Tricia McGinnity. We are grateful to those who have helped with recruitment, the NIHR Clinical Research Network Wessex and staff at the following hospitals: Birmingham Children's Hospital, Countess of Chester, Heartlands Hospital, Macclesfield District General Hospital, Manor Hospital, New Cross Hospital, Royal Bolton Hospital, Royal Manchester Children's Hospital, Royal Preston Hospital, Royal Stoke Hospital, Russell's Hall Hospital, St Mary's Hospital (Isle of Wight), Southampton General Hospital, University Hospital Coventry, Walsall Manor Hospital, Warwick Hospital, Whiston Hospital and Worcestershire Royal Hospital. Finally, we would like to thank all the participants, patients, parents and healthcare practitioners who have taken time to help us with this research.

Conflict of interest: R. Knibb reports grants from Asthma UK, during the conduct of the study. S. Latter reports grants from Asthma UK, during the conduct of the study. C. Liossi reports grants from Asthma UK, during the conduct of the study. F. Mitchell has nothing to disclose. R. Radley has nothing to disclose. G. Roberts reports grants from Asthma UK, during the conduct of the study. S. Holley reports grants from Asthma UK, during the conduct of the study.

Support statement: This study was supported by a research grant from Asthma UK (AUK-PG-2013-213). Funding information for this article has been deposited with the Crossref Funder Registry.

\section{References}

1 Fleming L, Murray C, Bansal AT, et al. The burden of severe asthma in childhood and adolescence: results from the paediatric U-BIOPRED cohorts. Eur Respir J 2015; 46: 1322-1333.

2 Thomas M. Why aren't we doing better in asthma: time for personalised medicine? NPJ Prim Care Respir Med 2015; 25: 15004 .

3 Holley S, Morris R, Knibb R, et al. Barriers and facilitators to asthma self-management in adolescents: a systematic review of qualitative and quantitative studies. Pediatr Pulmonol 2017; 52: 430-442.

4 Marks R, Allegrante JP, Lorig K. A review and synthesis of research evidence for self-efficacy-enhancing interventions for reducing chronic disability: implications for health education practice (part II). Health Promot Pract 2005; 6: 148-156.

5 Bandura A. Self-Efficacy in Changing Societies. Cambridge, Cambridge University Press, 1995.

6 Scherer YK, Bruce S. Knowledge, attitudes, and self-efficacy and compliance with medical regimen, number of emergency department visits, and hospitalizations in adults with asthma. Heart Lung 2001; 30: 250-257. 
7 Mancuso CA, Rincon M, McCulloch CE, et al. Self-efficacy, depressive symptoms, and patients' expectations predict outcomes in asthma. Med Care 2001; 39: 1326-1338.

8 Lavoie KL, Bouchard A, Joseph M, et al. Association of asthma self-efficacy to asthma control and quality of life. Ann Behav Med 2008; 36: 100-106.

9 Mancuso CA, Sayles W, Allegrante JP. Knowledge, attitude, and self-efficacy in asthma self-management and quality of life. J Asthma 2010; 47: 883-888.

10 Carpenter DM, Ayala GX, Williams DM, et al. The relationship between patient-provider communication and quality of life for children with asthma and their caregivers. J Asthma 2013; 50: 791-798.

11 Martin MA, Catrambone CD, Kee RA, et al. Improving asthma self-efficacy: developing and testing a pilot community-based asthma intervention for African American adults. J Allergy Clin Immunol 2009; 123: 153-159.

12 Bursch B, Schwankovsky L, Gilbert J, et al. Construction and validation of four childhood asthma self-management scales: parent barriers, child and parent self-efficacy, and parent belief in treatment efficacy. J Asthma 1999; 36: 115-128.

13 Rhee H, Belyea MJ, Ciurzynski S, et al. Barriers to asthma self-management in adolescents: relationships to psychosocial factors. Pediatr Pulmonol 2009; 44: 183-191.

14 Sleath B, Carpenter DM, Slota C, et al. Communication during pediatric asthma visits and self-reported asthma medication adherence. Pediatrics 2012; 130: 627-633.

15 Zebracki K, Drotar D. Outcome expectancy and self-efficacy in adolescent asthma self-management. Children's Health Care 2004; 33: 133-149.

16 Riekert KA, Borrelli B, Bilderback A, et al. The development of a motivational interviewing intervention to promote medication adherence among inner-city, African-American adolescents with asthma. Patient Educ Couns 2011; 82: 117-122.

17 Burbank AJ, Lewis SD, Hewes M, et al. Mobile-based asthma action plans for adolescents. J Asthma 2015; 52: 583-586.

18 Rhee H, McQuillan BE, Belyea MJ. Evaluation of a peer-led asthma self-management program and benefits of the program for adolescent peer leaders. Respir Care 2012; 57: 2082-2089.

19 van Dellen QM, Stronks K, Bindels PJ, et al. Adherence to inhaled corticosteroids in children with asthma and their parents. Respir Med 2008; 102: 755-763.

20 van Es SM, Kaptein AA, Bezemer PD, et al. Predicting adherence to prophylactic medication in adolescents with asthma: an application of the ASE-model. Patient Educ Couns 2002; 47: 165-171.

21 van Es SM, Nagelkerke AF, Colland VT, et al. An intervention programme using the ASE-model aimed at enhancing adherence in adolescents with asthma. Patient Educ Couns 2001; 44: 193-203.

22 Brod M, Tesler LE, Christensen TL. Qualitative research and content validity: developing best practices based on science and experience. Qual Life Res 2009; 18: 1263-1278.

23 Bandura A. Guide for constructing self-efficacy scales. In: Urdan T, Pajares F, eds. Self-Efficacy Beliefs of Adolescents. Vol. 5. Greenwich, CT, Information Age Publishing, 2006; pp. 307-338.

24 Frei A, Svarin A, Steurer-Stey C, et al. Self-efficacy instruments for patients with chronic diseases suffer from methodological limitations - a systematic review. Health Qual Life Outcomes 2009; 7: 86.

25 Orrell-Valente JK, Jarlsberg LG, Hill LG, et al. At what age do children start taking daily asthma medicines on their own? Pediatrics 2008; 122: e1186-e1192.

26 Schlösser M, Havermans G. A self-efficacy scale for children and adolescents with asthma: construction and validation. J Asthma 1992; 29: 99-108

27 Frei A, Svarin A, Steurer-Stey C, et al. Self-efficacy instruments for patients with chronic diseases suffer from methodological limitations - a systematic review. Health Qual Life Outcomes 2009; 7: 86.

28 Braun V, Clarke V. Using thematic analysis in psychology. Qual Res Psychol 2006; 3: 77-101.

29 Spirito A, Stark LJ, Williams C. Development of a brief coping checklist for use with pediatric populations. J Pediatr Psychol 1988; 13: 555-574.

30 Schwarzer R, Jerusalem M. Generalized Self-Efficacy Scale. In: Johnston M, Weinman J, Wright SC, eds. Measures in Health Psychology: a User's Portfolio. London, nferNelson, 1995; pp. 35-37.

31 DeVellis RF. Scale Development: Theory and Applications. Vol. 26. Los Angeles, CA, Sage Publications, 2016.

32 Edgecombe K, Latter S, Peters S, et al. Health experiences of adolescents with uncontrolled severe asthma. Arch Dis Child 2010; 95: 985-991. 